\title{
Complex networks and waveforms from acoustic emissions in laboratory earthquakes
}

\author{
H. O. Ghaffari ${ }^{1}$, B. D. Thompson ${ }^{2}$, and R. P. Young ${ }^{1}$ \\ ${ }^{1}$ Department of Civil Engineering and Lassonde Institute, University of Toronto, Toronto, 170 College Street, \\ M5S 3E3, ON, Canada \\ ${ }^{2}$ Mine Design Engineering, 1045 John Counter Boulevard, Kingston, ON K7K 6C7, Canada \\ Correspondence to: H. O. Ghaffari (h.o.ghaffari@gmail.com)
}

Received: 17 August 2013 - Revised: 21 May 2014 - Accepted: 10 June 2014 - Published: 24 July 2014

\begin{abstract}
Understanding the physics of acoustic excitations emitted during the cracking of materials is one of the longstanding challenges for material scientists and geophysicists. In this study, we report novel results of applications of functional complex networks on acoustic emission waveforms emitted during the evolution of frictional interfaces. Our results show that laboratory faults at microscopic scales undergo a sequence of generic phases, including strengthening, weakening or fast slip and slow slip, leading to healing. For the first time we develop a formulation on the dissipated energy due to acoustic emission signals in terms of shortterm and long-term features (i.e., networks' characteristics) of events. We illuminate the transition from regular to slow ruptures. We show that this transition can lead to the onset of the critical rupture class similar to the direct observations of this phenomenon in the transparent samples. Furthermore, we demonstrate the detailed submicron evolution of the interface due to the short-term evolution of the rupture tip. As another novel result, we find that the nucleation phase of most amplified events follows a nearly constant timescale, corresponding to the initial strengthening or locking of the interface. This likely indicates that a thermally activated process can play a crucial role near the moving crack tip.
\end{abstract}

\section{Introduction}

The 2011 Tohoku-Oki earthquake $\left(M_{\mathrm{w}}=9.0\right)$ showed how a slow-slip phenomenon can lead to destructive ruptures with emerging successive rupture transitions from slow to subRayleigh and super-shear ruptures (Meng et al., 2011; Maercklin et al., 2012; Simons et al., 2011). Similar dramatic tran- sitions from creep fronts or slow-slip events (SSE) to fast and sometimes critical ruptures have been reported in laboratory earthquakes (Rubinstein et al., 2004; Ben-David et al., 2010a, b; Ghaffari and Young, 2013; Nielsen et al., 2010).

Existing theoretical studies of rupture transitions have focused mostly on regular rupture to super shear (Andrews, 1976; Dunham, 2007; Rice et al., 2001).

Recently, a few numerical models have shown the evolution of creeping faults or SSE into critical earthquakes, using the coupling of friction laws with heat and pore pressure (Noda and Lapusta, 2013; Segall et al., 2010).

The assumption of a massive thermal energy release is a vital part of the employed coupled equations of the models. In these models, the weakening of the frictional-rock interface (i.e., fault) is obviously due to rapid shear heating of pore fluids and does not accord with the aforementioned laboratory friction experiments. Some other simpler models (Kaneko and Ampuero, 2011) - based on state and rate equations - capture essentials of rupture transitions in dry interfaces. However, these models are unable to predict all features of the laboratory observations. Thus, in the latter models, the real mechanism of velocity weakening or velocity strengthening in terms of friction evolution at micron and submicron scales is not known (Ben-David et al., 2010b).

In addition to these developments, recent precise laboratory measurements have presented new insights into rupture events with microsecond resolution (Ben-David et al., 2010b, c). Among these findings, universal trends of appropriate parameter spaces (such as displacement versus time or temporal contact area) were of considerable interest (Ben-David et al., 2010b). While the short-term evolution of ruptures (and then friction) can be demonstrated fairly well by universality of 
temporal strain (or velocity) profiles, the long-term evolution of an interface is characterized by the distribution of appropriate parameters. Among the long-term attributes, one can consider the distribution of waveform amplitudes, the distribution of waiting times between successive events and the long-term evolution of healing parameters (such as contact areas).

To support the aforementioned ideas, we have recently used complex network techniques to extract "hidden" information from recorded waveforms as well as reconstructed or recorded images through simple friction tests of glassy and rock samples (Ghaffari and Young, 2013). We showed that "functional" networks constructed over submicron events ("precursor or foreshocks" laboratory earthquakes) can unravel the possible regime of the rupture, while the changes of dynamic phases of network parameters are correlated with weak to very powerful events (cf. the energy spectrum of events) (Ghaffari and Young, 2013). Here, we explore the transition regimes of ruptures in Westerly granite-frictional interfaces. To this aim, we use several network parameter spaces, while the energy of the obtained networks (and the corresponding acoustic pulses) is expressed in terms of modularity profiles. Interestingly, we find that our formulation based on functional friction networks presents a solution to dramatic rupture transitions on a laboratory scale. Building on the evolutionary phases of modularity indices, we also present a new way of estimating fracture energy where a glass transition period is matched with a rapid evolutionary phase of module fast growth. Our results on the trends of fracture energy in different regimes of ruptures confirm recent theoretical and numerical studies regarding the physics of fracture energy. Furthermore, a slip-weakening model, constructed upon the frictional resistivity of the interfaces, is proposed in which the resistivity-slip rate phase space is related to our networks' attributes.

The organization of the paper is as follows: the second section, briefly, outlines our experiments on frictional rock interfaces. The third section reviews the proposed method to construct complex networks over recorded acoustic signals. The next part covers the features of networks, particularly, several parameter spaces and the study of the left-handed asymmetric shape of crackling noises. In this section, we show that the trend of crack-like ruptures in post-peak behavior follows a time-weakening mechanism with nearly exponential decay with time. Section 5 demonstrates the coupling of long-term and short-term networks' features of crackling noises, which reveals the full energy spectrum of events and their transitions. Section 6 introduces an effective temperature model for the fast-slip phase of a single event, while the results of the model explain the left-handed asymmetric shape of acoustic crackling noises. We show that for silicate rocks this phase has a shorter duration than for cement and poly methyl methacrylate (PMMA), indicating a direct relation of network parameters with fundamental mechanical parameters of materials. Finally, we conclude with our results in the last section and shed light on possible further applications of the discussed algorithm to other sources of acoustic excitations.

\section{Reviews of experiments}

The detailed experimental results have been reported in Thompson et al. (2005, 2009). Our main data set includes the recorded discrete and continuous waveforms of acoustic emissions (AEs) using 16 piezoelectric transducers from a saw-cut sample of Westerly granite (LabEQ1), under triaxial loading. The saw cut was at a $60^{\circ}$ angle and polished with silicon carbide 220 grit. Each triggered event had the duration of $204.8 \mu \mathrm{s}$ (recorded at $5 \mathrm{MHz}$ ) while the three main stick-slip events were occurring. The experiment was servo-controlled using an axial strain rate of $5 \times 10^{-6} \mathrm{~s}^{-1}$ ( $\sim 10 \mu \mathrm{m} \mathrm{s}^{-1}$ as the loading rate). The confining stress was maintained at $150 \mathrm{MPa}$ for three reported main stick-slip events, producing 109 located rupture fronts. The second data set (LabEQ2) contains two main cycles of loadingunloading (stick-slip) of Westerly granite on an embedded natural fault by loading at constant confining pressure. A natural rough fault was created using a triaxial loading system with constant confining pressure of $50 \mathrm{MPa}$ and acoustic emission feedback control.

\section{Functional friction networks}

Building networks over recorded time series from different recorded instruments (receivers or observers) has recently attracted great interest in the analysis of time series from brain activity (Bassett et al., 2006), climate (Donges et al., 2009), conformational dynamics of proteins ( $\mathrm{Li}$ et al., 2008) and physiology (Bashan et al., 2012). Many methods for analyzing the interactions between two or more time series have been proposed (Schiff et al., 1996; Arnhold et al., 1999; Chenet al., 2004; Napoletani and Sauer, 2008). In the case of single records, recurrence networks as new tools to analyze time series have been used in many fields of research (Donner et al., 2010). A recently proposed network's meta-time-series considers the power of the multi-observation of an event or series of events through different observatories (Iwayama et al., 2012). We recently employed a similar (but simpler) meta-time-series analysis on the quasi-static evolution of apertures and real-time contact areas (Ghaffari and Young, 2012a, b). Here, we describe the construction of a network on each time step in which the nodes correspond to acoustic sensors where any elastic excitement induces voltagefluctuations in them. We show that the nature of piezoelectric signals can be quantified through this new tool, resembling main features of instabilities at submicron scales. We note that these functional friction networks are not physical networks; however the underlying functionality of constructed networks correlates with the main mechanical features of 
friction instabilities. We use the word "functional" to distinguish our networks from the possible ones constructed over particles' interactions (such as force networks in granular materials).

\subsection{Network construction}

Here we review the previously introduced algorithm (Ghaffari and Young, 2013). This algorithm includes the following five steps:

1. The waveforms recorded in each station are normalized.

2. Each time series is divided according to maximum segmentation, in a way that each segment includes one and only one data point. We consider each recorded point in each waveform with the length of $t_{\max }=204 \mu \mathrm{s}$. The $j$ th segment from $i$ th time series $(1 \leq i \leq N)$ is denoted by $x^{i, j}(t) . N$ is the number of nodes or acoustic sensors. We put the length of each segment as $a$ unit. This essentiality considers the high temporal resolution of the system's evolution, smoothing the raw signals with 20-60 time windows (for Lab.EQ1 \& 2 it is equal to $1-3 \mu$ s).

3. $x^{i, j}(t)$ is compared with $x^{k, j}(t)$ to create an edge among the nodes. If $d\left(x^{i, j}(t), x^{k, j}(t)\right) \leq \xi$, we set $a_{i k}(j)=1$ otherwise $a_{i k}(j)=0$, where $a_{i k}(j)$ is the component of the connectivity matrix and $d(\cdot)=$ $\left\|x^{i, j}(t)-x^{k, j}(t)\right\|$ is the employed similarity metric.

4. Threshold level $(\xi)$ : to select a threshold level, we use betweenness centrality (B.C) - see Eq. (1) below for the definition of this property. In Fig. 1d of Ghaffari and Young (2012b), we showed that selecting the threshold level by using the minimum variation of B.C versus $\xi$ is equivalent with finding the most stable structures in networks. We note that the truncation level (or an interval of that) determines the characteristics of the network. If it is extremely small, the nodes with weak similarity are also connected ("fake connection"). As the value of $\xi$ increases, the number of links becomes smaller. Since friction networks are dynamic networks, we prefer to use a dynamic threshold parameter rather than a constant threshold level.

5. Increase the resolution of visualization: knowing that functional networks generally reveal a good performance for a small number of nodes (Donges et al., 2009), we increased the size of the adjacency matrix with the simple interpolation of $d$ using cubic spline interpolation. Further analysis shows that the method is nearly insensitive for $N>15$. The increase in the number of nodes to 50 generally did not change the results presented below for the acoustic-friction networks and merely increased the visual quality of the results.

\subsection{Network parameters}

In this work, we apply some of key network measures. We will use some of these metrics to introduce phase diagrams and for further interpretation of obtained results. Each node is characterized by its degree $k_{i}$ representing the number of links, and its B.C (Newman and Girvan, 2004):

$$
\text { B.C }_{i}=\frac{1}{(N-1)(N-2)} \sum_{\substack{h, j \\ h \neq j, h \neq i, j \neq i}}^{N} \frac{\rho_{h j}^{(i)}}{\rho_{h j}},
$$

in which $\rho_{h j}$ is the number of shortest paths between $h$ and $j$, and $\rho_{h j}^{(i)}$ is the number of shortest paths between $h$ and $j$ that pass through $i$. To describe the correlation between the degree of a node and the degrees of neighboring nodes, the assortativity, $r_{k}=\frac{\left.<j_{1} k_{1}>-<k_{1}\right\rangle^{2}}{\left.<k_{1}^{2}>-<k_{1}\right\rangle^{2}}$, is defined as the Pearson correlation coefficient of the degrees at both ends of an edge. $j_{1}$ and $k_{1}$ refer to the node degrees at both ends of the same degree. Here, $\langle\ldots\rangle$ denotes average over the number of links in the network (Newman and Girvan,2004).

The network's modularity characteristic is addressed as the quantity of densely connected nodes relative to a null model (random model). The main diagnostic in this work is the $Q$ profile. The modularity is the result of some optimization of the cluster structure of a given network. The modularity $Q$ (i.e., objective function) is defined as (Guimerà and Amaral, 2005; Newman, 2010):

$Q=\sum_{\mathrm{s}=1}^{N_{\mathrm{M}}}\left[\frac{l_{\mathrm{s}}}{L}-\left(\frac{d_{\mathrm{s}}}{2 L}\right)^{2}\right]$

in which $N_{\mathrm{M}}$ is the number of modules (clusters), $L=$ $\frac{1}{2} \sum_{i}^{N} k_{i}, l_{\mathrm{s}}$ is the number of links in module "s" and $d_{\mathrm{s}}=\sum_{i} k_{i}^{\mathrm{s}}$ (the sum of node degrees in module s). We use the Louvian algorithm to optimize Eq. (2) (Blondel et al., 2008), which has been used widely to detect communities in different complex networks. Then, in each time step during the evolution of waveforms (here $\sim 200 \mu$ s), we obtain a $Q$ value. The temporal evolution of $Q$ values in the monitored time interval forms the $Q$ profile. $Q$ profiles carry generic universal timescales, likely distinguishing microsecond-scale details of microcracks. Regarding the wide range of applications of acoustic emissions and their observation in an extraordinarily large number of experiments, finding such a universal frame on complicated emitted waveforms is very important. This approach likely presents a road to draw a universal picture of acoustic emission sources, close to the mechanisms of breaking or deforming atomic bonds.

Finally, the number of triangles centered at a node $i$ in the constructed networks, denoted by $T_{i}$, is used to define the clustering coefficient: $c_{i}=\frac{2 T_{i}}{k_{i}\left(k_{i}-1\right)}$. Based on the introduced measures several parameter spaces will be employed 

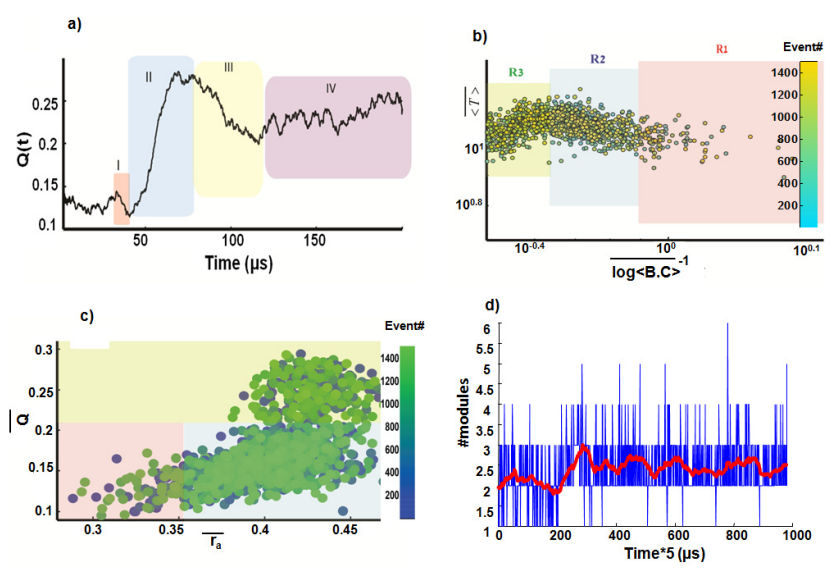

Figure 1. Evolution of rupture fronts in a rough fault. (a) $Q$ profile resulting from the mapping of acoustic waveforms from a regular event, including the four main evolutionary phases (Ghaffari and Young, 2013; Ghaffari et al., 2014). Two sharp transitions from phase I to II and from phase II to phase III. (b) The scaling of $\overline{\log <\text { B.C }}^{-1}$ with the temporal average of the mean number of triangles per node $(\overline{\langle T\rangle})$ differentiates three main clusters: $\overline{\langle T\rangle} \propto \overline{\log <\text { B.C }>}^{-\chi}$ (see also Fig. 2a), and (c) higher time average assortativity scales with higher modularity value, with a separation of events with high modular values, characterizing slow-slip ruptures. The colors in (b) and (c) show the events' sequences, corresponding to time. (d) Evolution of number of communities for a regular event. The blue and red profiles are raw and smoothed data points, respectively. We have used Levenberg-Marquardt algorithm to define the best fits.

to analyze the structures and the possible physical mechanisms behind the obtained networks. Parameter spaces such as $c-k, C-$ B.C (or $k-$ B.C) have been previously used widely in studying a wide range of networks (Donges et al., 2009; Ghaffari et al., 2013; Guimerà and Amaral, 2005).

\section{Network parameter spaces of AEs events}

Our algorithm results in a typical $Q$ profile for each single event (Fig. 1a). This profile presents some basic features of a single event as the result of the breaking of an asperity. In the following sections, we show how the temporal evolution of the modularity index represents generic dynamics of a single asperity failure, including information on nucleation, fastdeformation and fast-slip parts. Inspecting over 8000 events from Lab.EQ1 and Lab.EQ2 reveals that the general evolution of $Q$ profiles is universal, imprinting nearly constant timescale characteristics for each evolutionary phase (Ghaffari and Young, 2013; Ben-David et al., 2010c). The main evolutionary phases, which can be seen from Fig. 1a, are as follows: phase I is characterized by a rapid drop of modularity reaching the overall minimum value of $Q(t)$. Considering that the laboratory earthquakes are in near field, approaching the crack front, the deformation or crushing of the "node" or asperity is the possible explanation for this stage. The duration of this phase is $4-8 \mu \mathrm{s}$. We note that this phase corresponds to an initial strengthening of fault, which imposes an obstacle for rupture growth. The duration of this phase is much shorter than the other following generic phases. The sudden release of energy - stored during a few microseconds - likely dictates a rupture dimension and rupture regime. Later, we will use the inverse of the $Q$ profiles to magnify the first phase as the nucleation-deformation phase.

The second phase is characterized by a fast growth of modularity (Fig. 1a, d) and increase in the number of modules (Fig. 1d). As we have shown in Ghaffari and Young (2013), the rate of this rebounding phase is scaled with the maximum value of modularity. The collapsed data set for this phase indicates a universal generic dynamic, which is confirmed by a constant value of the power-law coefficient $\alpha_{2} \approx 1.5 \pm 0.3$ for both Lab.EQ1 and Lab.EQ2 (see Supplement of Ghaffari and Young, 2013). The duration of this phase is 25$35 \mu$ s. The possible mechanism beyond this phase is the fast slip of the interface, which is arrested or damped at the end of the phase. It is important to understand why the duration of this phase is nearly constant for both experiments while the general loading conditions are different. We will show in Sect. 6 that this timescale is different in cement (concrete). Then, we infer that the fast-weakening phase is a characteristic of tiny ruptures with strong dependency on the molecular/atomic structure of materials. Assuming a velocity range of $5-500 \mu \mathrm{m} \mathrm{s}^{-1}$ for phase II, we obtain a maximum displacement of $\sim 150 \mathrm{pm}-15 \mathrm{~nm}$. This simple calculation shows that tiny-amplified events are closely related to the failure of the atomic bonds of heterogeneities and asperities. From this perspective, emitted noise from the failure of chemical bonds in the vicinity of a crack tip should be strongly scaled with the molecular structure and forces between them (leading to cohesive forces or surface energy). The maximum displacement at the end of phase II changes to $1-5 \mu \mathrm{m}$ if the frictional slip rate increases to $50-100 \mathrm{~mm} \mathrm{~s}^{-1}$ (the reported value for PMMA in Ben-David et al., 2010c). This shows that the recorded precursor events encode a displacement magnitude at the range of pico- to nanometers (and at most micrometers), demonstrating possible nanoearthquakes in terms of displacement of the interface.

The transition to the last evolutionary phase (phase IV) which results in a decreasing modularity is accompanied by an unusual gradual decay of the communities (phase III). We will use a theory of glass transition employing an effective temperature model to explain the emergence of this phase (see Sect. 6). The last stage is generally a decaying phase, indicating a slow-slip phase. The rate of the slow-slip phase, $\dot{Q}_{\text {IV }}$, scales with the maximum recorded modularity at a rate $\left|\dot{Q}_{\mathrm{IV}}\right| \propto Q_{\max }^{\lambda}$ where $\lambda$ is nearly constant for the regular events (see Supplement of Ghaffari and Young, 2013). Regular events are events with a rupture speed below a threshold level (Rayleigh velocity). Furthermore, a rough scaling between the rate of the first phase and the last phase was 

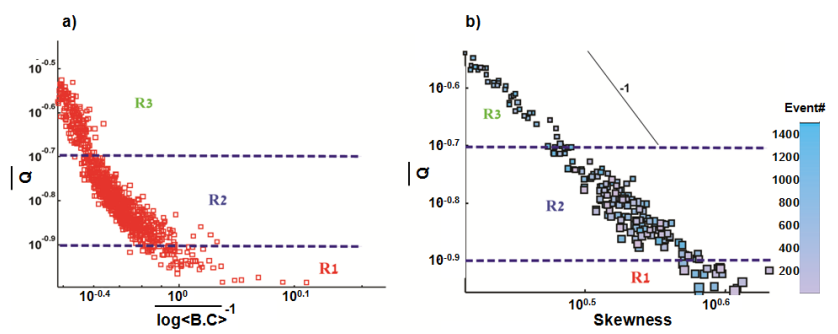

Figure 2. Asymmetric nature of $Q$ profiles from LabEQ2. (a) $\overline{\log <\text { B.C }>}{ }^{-1}-\bar{Q}$ plane with three distinct classes. (b) The average skewness of the $Q$ profiles scales with $\bar{Q}$, indicating a smaller positive skewness of pulse shape for events from the R3 class. The size of the squares corresponds to the intensity of $\overline{\log \langle\text { B.C }\rangle}-1$; colors show the sequence of events.

found (Ghaffari and Young, 2013): $\left|\dot{Q}_{\mathrm{IV}}\right| \propto\left|\dot{Q}_{\mathrm{I}}\right|^{-\gamma}$, where $\gamma$ is slightly larger for slow-slip events. Note that formal hypothesis tests and alternative models are not applied here due to the small network size. To get a spatiotemporal evolutionary picture of the recorded events (and then relatively longterm evolutionary patterns of ruptures), we use the mean values of network diagnostics. Applying this idea to Lab.EQ1 and Lab.EQ2, we recognized several correlations among network attributes (Figs. 1b, c and 2a).

The parameter spaces shown in Fig. $1 \mathrm{~b}$ and c clearly display three main classes of events. In Fig. 2a, we show one of the parameter spaces for Lab.Eq.2: $\frac{\overline{\log }\left\langle\text { B.C }^{-1}-\right.}{2}$ $\bar{Q}$. Hereafter, $\langle\ldots\rangle$ refers to the average over all nodes, whereas the bar sign denotes averaging over time. For rel-

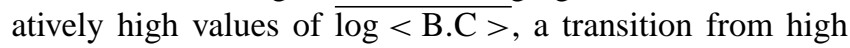
modularity to intermediate modularity is observed. For the saw-cut experiment, we found a unique separation of some events, providing a slow deformation (R3 class) with respect to the duration of phase I and a similarity to the phase IV (Ghaffari and Young, 2013). A similar transition is observed in $\overline{\langle T\rangle}-\overline{\log \langle\text { B.C }\rangle}-1$ and $\bar{Q}-\overline{r_{\mathrm{a}}}$ (Fig. 1b, c), in which $\overline{\langle T\rangle}$ is the spatiotemporal mean number of triangles centered at each node. We recognize three main clusters of events: R1, R2 and R3. In this classification, the trends of parameter values within the clusters are considered. $\mathrm{Ob}$ viously, one can also use simple clustering methods such as K-means clustering, a self-organizing feature map or other data-clustering algorithms (Ghaffari et al., 2013). The R1 phase contains events with relatively high energy, while regular events with intermediate energy are assigned to the R2 class. The R3 class has a longer evolutionary phase I and represents longer and (on average) weaker events, indicating a slow-slip phenomenon. Based on Figs. 1b and 2a we infer approximate power laws describing the scaling of network properties for the recorded events: $\overline{\langle T\rangle} \propto \overline{\log <\text { B.C }\rangle}-\chi$ and $Q_{\max } \propto \Xi^{-\pi}$, in which $\Xi=\overline{\log <\text { B.C }>}-1$, and $\pi$ and $\chi$ are nearly constant exponents for each class. Obviously, the transition from the $\mathrm{R} 3$ to the $\mathrm{R} 2$ class is accompanied by a change of the sign of $\chi$ from positive to negative.

Furthermore, we investigate the asymmetric nature of crackling-noise pulses in terms of $Q$ profiles. The average skewness of $Q$ profiles as an asymmetry measure can be quantified by (Eq. 1 in Zapperi et al., 2005)

$\Sigma=\frac{\frac{1}{t_{\max }} \int_{0}^{t_{\max }} Q(t)(t-\hat{t})^{3} \mathrm{~d} t}{\left[\frac{1}{t_{\max }} \int_{0}^{t_{\max }} Q(t)(t-\hat{t})^{2} \mathrm{~d} t\right]^{3 / 2}}$,

in which $\hat{t}=\frac{1}{t_{\max }} \int_{0}^{t_{\max }} Q(t) t \mathrm{~d} t$ is the modularity-weighed mean and $t_{\max }=204 \mu \mathrm{s}$. In Fig. $2 \mathrm{~b}$, we have compared the skewness with the mean modularity for the Lab.EQ2 events.

The data show an inverse correlation of the leftward asymmetric shape of the acoustic waveforms with the regime of ruptures indicated by $\bar{Q}$. This is a universal feature of "crackling-noise" systems which have been allocated to the nature of the dissipation energy, such as eddy currents in Barkhausen noise (movement of magnetic domain wall) or threshold strengthening in moment rate profiles in natural earthquakes (Mehta et al., 2002; Zapperi et al., 2005). We conclude that a large deviation from symmetry is the signature of ruptures with relatively high energy and critical ruptures, while approaching a less asymmetric shape indicates ruptures with lower energy. Then, understanding the details of the microsecond-scale evolution of $Q$ profiles helps to evaluate the general shape of crackling noise.

Next, to understand the role of the main deformation phase I, we define a parameter of resistivity against motion: $R \equiv$ $\frac{1}{Q_{\text {norm }}}$, where $Q_{\text {norm. }}=\frac{Q}{Q_{0}}\left(\mathrm{Q}_{0}\right.$ is the equilibrium value of $\bar{Q}(t))$. In Fig. 3a, we show the $R$ profiles from Lab.EQ1. In Fig. 3c, we also display the distribution of events from Lab.EqQ1 in a normalized space: $(\dot{R})_{I}-R_{\max }$, indicating a preferred timescale of $\sim 2-5 \mu$ s for the first phase and for most of the events.

Approaching the critical stage and subsequent fast-slip phase can be quantified by the parameter $S_{R}=\frac{R_{\max }-R_{0}}{R_{0}-R_{\min }}$ (Fig. 3a inset). This parameter is similar to the seismic $S$ factor: $S_{\mathrm{s}}=\frac{\tau_{\mathrm{p}}-\tau_{0}}{\tau_{\mathrm{o}}-\tau_{\mathrm{r}}}$ (Andrews, 1976; Dunham, 2007; Rice et al., 2001), which is defined over shear strength variations during a period of shear displacement, where $\tau_{\mathrm{p}}, \tau_{0}$ and $\tau_{\mathrm{r}}$ are the maximum, initial and steady-state shear strengths, respectively. It has been widely discussed (Andrews, 1976; Dunham, 2007) that the value of $S_{\mathrm{s}}$ could be used to determine the regime of rupture; for instance it has been shown that a super-shear rupture may occur below a critical value of $S_{\mathrm{s}}<S_{\mathrm{c}}$ (Andrews, 1976). In other words, with increasing $S_{\mathrm{S}}$ the probability of "critical ruptures" decreases, and the regime of the rupture approaches regular and slow ruptures. In Fig. 3d, we show that $S_{R}$ scales with $Q_{\max }$, representing ruptures with smaller $Q_{\max }$ that hold higher $S_{R}$. On the other hand, generally smaller $Q_{\max }$ indicates weaker and slower failures at submicron scales. Consequently, we can use $S_{R}$ for a possible classification of rupture fronts. 

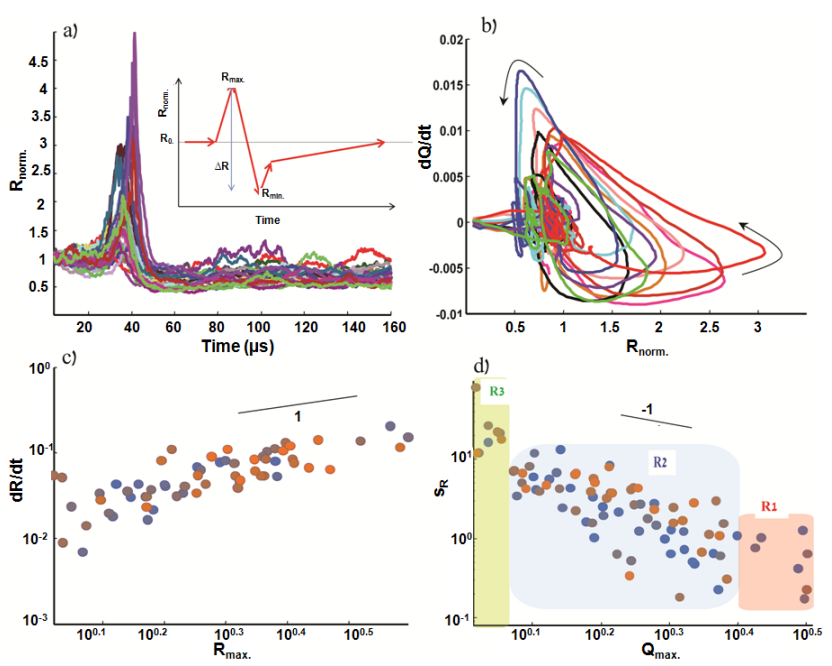

Figure 3. "Resistivity" against interface motion and the onset of slip weakening at microsecond resolution. (a) The reciprocal of $Q$ profiles is used to magnify the evolution of the first phase. We assume that the resistivity against the slip is inferred from the reciprocal $Q$ values. We show $\sim 30$ arrested rupture fronts from Lab.EQ1 and their normalized resistivity parameter (inset: a simplified form of the resistivity profile parameters to illustrate the definition of $S_{R}$ ). (b) Events in $R_{\text {norm. }}-\dot{Q}$ plane. (c) A nearly constant exponent $(\mu)$ in $\dot{R} \propto R_{\text {max }}^{\mu}$ represents a generic dynamics of the first evolutionary phase, indicating a failure or deformation timescale. (d) Events in $S_{R}-Q_{\max }$ parameter space indicate that events with $Q_{\max }$ show smaller $\mathrm{S}_{R}$ value (events from Lab.EQ1). In (c) and (d), the colors show the sequence of events. Earlier events are blue and the final ones are orange. Slopes of 1 and -1 for the coefficients of approximate power laws between the shown parameters are displayed for reference.

The $R_{\text {norm }}-\dot{Q}$ plane reflects a similar accelerationdeceleration phenomenon in frictional interfaces under high velocity (Fig. 3b) (Sone and Shimamoto, 2009). Here $R_{\text {norm. }}\left(R_{\text {norm. }}=\frac{R}{R_{0}}\right)$ is the normalized value of the resistivity profile and $R_{0}$ is the equilibrium value of $R(t)$. To fit a model in the $R_{\text {norm }}-\dot{Q}$ plane (Figs. $3 \mathrm{~b}$ and 4 ), we use the fact that the duration of the second phase ("fast slip" or slip weakening) is nearly constant for "regular" ruptures. In addition to this, to fit a proper function we use studies in which the slip rates of frictional interfaces are related to the frictional resistivity and also involve the acceleration and deceleration of faults during fast-slip rates (such as Eqs. 1 and 2 in Sone and Shimamoto, 2009). This leads to

$R=R_{0}+A\left(R_{\mathrm{p}}-R_{0}\right) \exp \left[\ln (0.03) t / t_{\mathrm{c}}\right]$,

in which $R_{0}(\dot{Q})=\exp \left(-\dot{Q} / \dot{Q}_{\mathrm{c}}, R_{\mathrm{p}}\right.$ is the maximum of resistivity and $t$ is time. We find that $t_{\mathrm{c}} \approx 70 \mu \mathrm{s}, \dot{Q}_{\mathrm{c}}=0.004$ and $A=4$ approximate most of the recorded experimental results reasonably well (Fig. 4). The presented relation essentially involves two main parameters: $R_{\mathrm{p}}$ and $t$. Considering that $t_{\mathrm{c}}$ includes some dead time and the first-phase duration in $Q$ profiles, we estimate that $\left(t_{\mathrm{c}}\right)_{\text {real }} \approx t_{\mathrm{II}}$, i.e., weakening

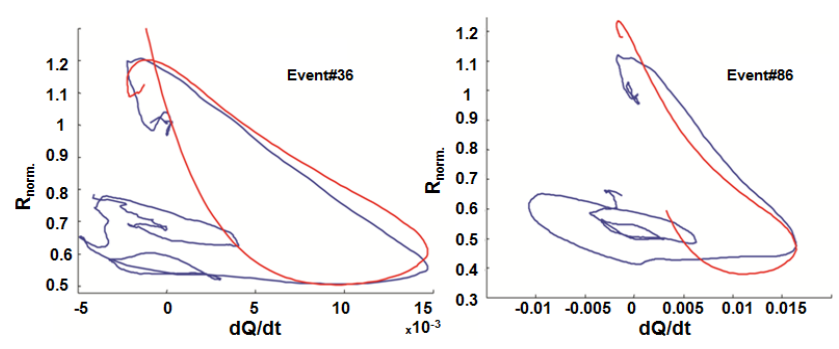

Figure 4. Comparing Eq. (4) with the experimental data from Lab.EQ1. A comparison of the phase space of precursor rupture fronts from smooth-rock interfaces in terms of the rate of $Q$ profiles and resistivity between experimental data (blue) and modeled friction (red).

time or fast-slip duration. As Eq. (4) is in time domain, however, it presents a quite similar phase space to $\mu_{\text {friction }}-\dot{u}$ (friction-slip rate) in Sone and Shimamoto (2009). We rationalize this similarity to micron and submicron rupture fronts when considering a time-weakening constitutive law, which prescribes that a fault's resistance weakens with increasing time over a characteristic timescale (see Andrews, 1985, for more information).

\section{Rupture energy based on modularity rate}

In this section, we use some of the previously derived relations to establish a possible link with the energy of ruptures. Moreover, we estimate fracture energy based on the revealed evolutionary phases' durations. In fact, this assumption implies that acoustic energy release during a rupture phenomenon may be assumed to be proportional to the dissipated energy. We develop an energy term for the fixed monitored time interval based on the rate of modularity evolution. We assume that a friction network and its energy evolve during a time interval and through the aforementioned distinct phases. We investigate the accumulation of energy of the system by calculating the rate of variation of communities in each generic phase. Then, we combine the shortterm parameters of an event (i.e., $Q$ profile) with the longterm trends of distinct events. We also speculate that the energy of functional networks in terms of the evolution of communities can be purely expressed in terms of three-point closed loops (triangles). In Xu et al. (2008), Milo et al. (2002) and Alon (2007), an analysis of motifs of different networks proved that the functionality of networks was correlated with the motif profiles. If the energy of a system could be expressed in terms of motifs, we can expect that the motif's frequencies are associated with the energy spectrum of the system. Here we do not extend our calculations to other types of motifs (such as open triangle or different four-point motifs). However, we conjecture that finding proper relations between motifs and other network parameters can provide similar (but not identical) expressions of the energy of the 
system. It is noted that the energy of networks has been studied previously, where an energy term was derived based on vertex degrees, global properties (such as the sizes of network components) and number of modules (Palla et al., 2004).

In Table A1, we summarize the parameters employed in our calculations. Considering $\overline{\langle T\rangle} \propto \overline{\log <\mathrm{B} . \mathrm{C}\rangle}^{-\chi}$, $\left|\dot{Q}_{\mathrm{IV}}\right| \propto Q_{\max }^{\lambda}$ and $Q_{\max } \propto \Xi^{-\pi}$ with $\Xi=\overline{\log <\mathrm{B} . \mathrm{C}>}^{-1}$, we find $\overline{\langle T>} \propto\left|\dot{Q}_{\mathrm{IV}}\right|^{\frac{-\chi}{\pi \lambda}}$. We approximate the kinematic energy of the system in terms of the energy of each evolutionary phase (shown in Fig. 1a) in $Q$ profiles: $E_{\text {system }} \approx$ $E_{\mathrm{I}}+E_{\mathrm{II}}+E_{\mathrm{III}}+E_{\mathrm{IV}}$. We also assume $E_{\mathrm{IV}} \propto \dot{Q}_{\mathrm{IV}}^{2}$, which leads to $E_{\mathrm{IV}} \propto<\bar{T}>\frac{-2 \pi \lambda}{\chi}$. This assumption is due to the similarity of $Q$ profiles to the recorded strains (normalized displacements) (Ben-David et al., 2010b). Ignoring the third phase, we estimate $E_{\mathrm{I}} \propto \dot{Q}_{\mathrm{I}}^{2}$ and get the first and the fourth contributions the energy by using $\left|\dot{Q}_{\mathrm{IV}}\right| \propto\left|\dot{Q}_{\mathrm{I}}\right|^{-\gamma}$, leading to $E_{\text {system }} \widetilde{\alpha} \overline{<T} \frac{-2 \pi \lambda}{\chi}\left(1+\overline{<T>} \frac{\frac{(2 \pi \lambda)(1+\gamma)}{\chi}}{<T}+E_{\mathrm{II}}\right)$. The last term (the second term in $E_{\text {system }}$ ) is expressed $E_{\mathrm{II}} \propto \dot{Q}_{\mathrm{II}}^{2} \propto<$ $\bar{T}>^{\frac{-1}{2 \times \pi \alpha_{2}}}$, where $\alpha_{2}$ is a constant for most of the events. Given these considerations, we find

$E_{\text {system }} \propto<\bar{T}>\frac{-2 \pi \lambda}{\chi}\left(1+<\bar{T}>^{\frac{(2 \pi \lambda)(1+\gamma)}{x}}\right)+<\bar{T}>^{\frac{-1}{2 \chi \pi \alpha_{2}}}$,

in which $\pi, \lambda, \gamma, \alpha_{2}>0$, representing the kinematic energy of the pulse in terms of $Q$ profiles. In other words, the energy of the waveforms is expressed by motif frequency and some exponents.

When $\chi<0$ (R2 regime in Fig. 1b), increasing the number of triangles leads the system to a new energy level as the minimum upper bound of R2: $E_{\text {system, } \chi<0}^{<\bar{T}>\rightarrow \max } \propto<\bar{T}>\frac{-2 \pi \lambda}{\chi}$ $+<\bar{T}>^{\frac{-1}{2 \chi \pi \alpha_{2}}}$. We denote this upper bound as the transition point from R3 to R2 (Fig. 5a). In other words, we have found that the kinematic energy at the transition point (R3 to R2 and vice versa) changes dramatically, resembling a first-order transition. The obtained result is similar to the direct observation of the transition from sub-Rayleigh to slow-rupture mode (Rubinstein et al., 2004; Ben-David et al., 2010a, b; Ghaffari and Young, 2013; Nielsen et al., 2010). Approaching $\chi=0$ has another interpretation, comparable with reaching the R1 (critical ruptures) class. Crossing the energy barrier at the singularity of this model can be interpreted as the generation of a high-energy rupture, i.e., a signature of $\mathrm{R} 1$ class. This interpretation is similar to the observation of super-shear ruptures in PMMA (reported in Rubinstein et al., 2004). In Rubinstein et al. (2004), it has been experimentally shown that acceleration of sub-Rayleigh rupture fronts towards Rayleigh velocity bifurcates into two different fronts: slow ruptures and super-shear ruptures.

Assuming the independence of scaling exponents from each other and approaching $\lambda \rightarrow 0$, the R3 class vanishes (Fig. 5a, green line). We conclude that $\lambda, \pi$ and $\gamma$ control transitions from R2 to R1 or R3, the sharpness of the transition from R2 to other classes and the evolution of R3 class, respectively. Remarkably, the transition from R2 to R1 (or R3) when approaching $\chi \geq 0$ shows a transition from a minimum $E$, indicating a jammed regime of the rupture. The jammed state of the rupture is important to understand in terms of network parameters because it can arrest the rupture as it propagates in the energy landscape. The same behavior is observed when $\gamma \rightarrow 0$, where the transition from R3 to $\mathrm{R} 1$ is trapped in a jammed state (not shown). Moreover, using the results presented in Ghaffari and Young (2013), we connect $\overline{\langle T\rangle}$ to the remote stress (strain) field. To this end, we use $\bar{Q} \propto \exp \left(-\kappa F_{\text {axial }}\right)$ and $\bar{Q} \propto \Xi^{-\eta}$, which yields $\log \langle T\rangle \propto \frac{\chi \kappa}{\eta} F_{\text {axial }}$, where $\kappa>0$ and $\eta$ are scaling exponents. $F_{\text {axial }}$ represents the external axial loading stress as the driving force (boundary condition). For the R2 class $(\chi<0)$, decreasing $\overline{\langle T\rangle}$ corresponds to an increasing external remote stress field, while the trend of $\overline{\langle T\rangle}$ is similar to $F_{\text {axial }}$ for $\chi>0$. To make use of this scaling relation, we substitute $\log \frac{\chi}{<T>} \propto \frac{\chi \kappa}{\eta} F_{\text {axial }}$ in the energy term, yielding

$E_{\text {system }} \widetilde{\propto}\left(\frac{\chi \kappa}{\eta} F_{\text {axial }}+1\right)^{\frac{2 \pi \lambda \gamma}{\chi}}+\left(\frac{\chi \kappa}{\eta} F_{\text {axial }}+1\right)^{\frac{-1}{2 \chi \pi \alpha_{2}}}$.

In the next step, we summarize two regimes for the slowfronts class (R3): (1) when $\chi$ is high, $E_{\text {system }}^{R 3} \rightarrow$ const, and (2) when $\chi$ is small or $F_{\text {axial }}$ is high, $E_{\text {system }}^{R 3} \propto$ $\left(\frac{\chi \kappa}{\eta} F_{\text {axial }}\right)^{\frac{2 \pi \lambda \gamma}{\chi}}$. It is noteworthy that in the presented results, $\chi$ is treated as the order parameter.

\section{Effective temperature model for microcracks}

In this section, we use an approach suggested for the (average) left-hand asymmetric shape of avalanches in cracklingnoise systems. Based on this approach (Mehta et al., 2002; Zapperi et al., 2005), the asymmetric average shape of the avalanches is due to the role of the energy dissipation process (the eddy currents in Barkhausen noise and the strengthening threshold in seismic moment signals). Here, we use a variant of the energy dissipation phenomenon in frictional interfaces, originally proposed in Ben-David et al. (2010c) to explain the abnormal drop of the phase III (Fig. 1a). The interpretation is as follows: a fast short-time fracturing (phase I) induces a very fast increase in the temperature of a tiny "process zone", which cools within a typical time. The main component of the theory is that the whole fracture energy is transferred to heat in the process zone. This can be assumed as an effective temperature approach, well described in annealing-embrittlement phenomena (Rycroft and Bouchbinder, 2012). Based on this theory, the annealing period is reflected in the form of a fast-slip phase, and the immediate strengthening stage is embrittlement (i.e., phase III in $Q$ profile). The increased temperature with respect to a reference 

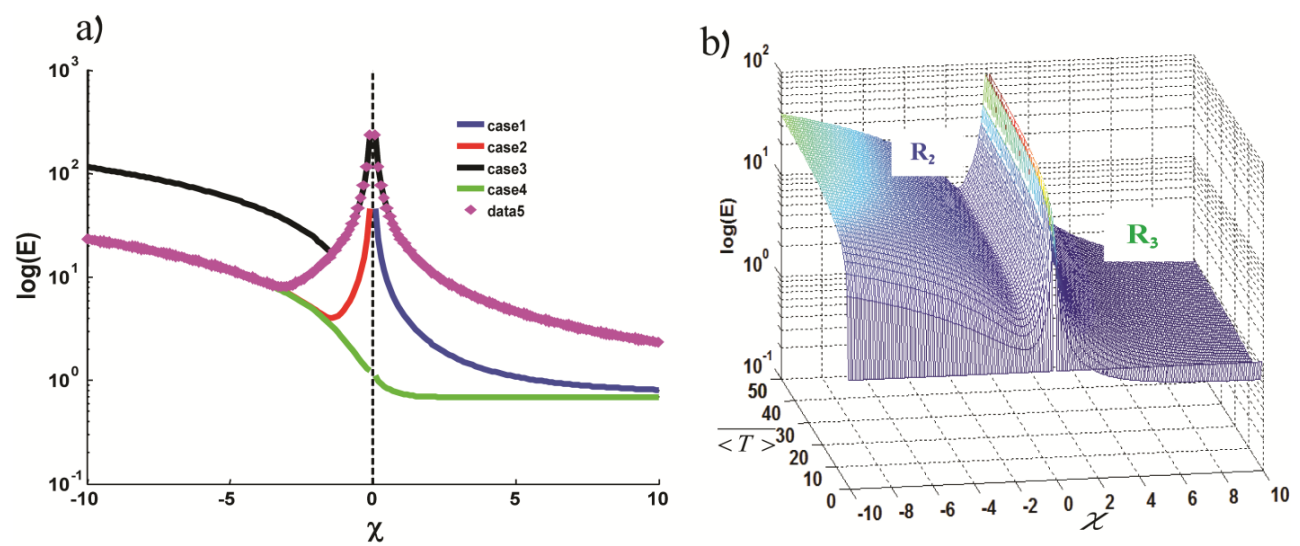

Figure 5. Rupture transition using the energy of $Q$ profiles. (a) Variation of the energy with the exponent in $\overline{<T>} \propto \overline{\log <\text { B.C. }>}-1$ shows a singlularity around $\chi=0$ in which a transition from $R_{3}$ to $R_{2}$ occurs for $\overline{\langle T}=10$. Case $1: \pi=\lambda=\gamma=1, \alpha=2$; case 2 : $\pi=\lambda=1$, $\gamma=5, \alpha=2$; case 3: $\pi=2, \lambda=\gamma=1, \alpha=2$; case 4: $\pi=\gamma=1, \lambda=0.01, \alpha=2$; case $5: \pi=\gamma=1, \lambda=5$, $\alpha=2$. (b) Dependence of the energy on $\pi$ and $\overline{<T>}$ for case 1 .

temperature (room temperature) for the 1-D case with imminently released energy as the source is given by Ben-David et al. (2010c):

$\Delta T_{\text {temp. }}=\frac{-\Gamma}{4 \rho c_{\mathrm{p}} h}\left[\operatorname{erf}\left(\frac{-h}{\sqrt{4 D_{\mathrm{T}} t}}\right)-\operatorname{erf}\left(\frac{h}{\sqrt{4 D_{\mathrm{T}} t}}\right)\right]$,

in which $h$ is the thickness of the process zone in which the energy rapidly dissipates, $D_{\mathrm{T}}$ is the thermal diffusivity, $\Gamma$ is the energy released in phase I (or fracture energy) and $t$ is the cooling time. As a first-order approximation, we estimate $t$ as follows:

$t \approx \frac{\Gamma^{2}}{\Theta}, \Theta=4\left(\Delta T \rho c_{\rho}\right)^{2} \pi D_{\mathrm{T}}$,

where $\Theta$ is a constant value for a given material. From the previous section, we assume $t \approx t_{\mathrm{II}}$. Since $\dot{Q}_{\mathrm{II}} \propto \frac{1}{t_{\mathrm{II}}}$, we discover $\dot{Q}_{\mathrm{II}} \propto \frac{\Theta}{\Gamma^{2}} \Rightarrow E_{\text {system }}^{\mathrm{II}} \propto \Gamma^{-4}$. Then $E_{\text {system in- }}$ directly includes the fracture energy. The implication of the obtained relation is remarkable when we analyze the fracture energy variation while considering the rupture velocity regimes: $E_{\text {system }}^{\mathrm{II}} \propto \overline{\langle T\rangle}^{-1 / 2 \chi \pi \alpha_{2}}$, yielding $\Gamma \propto$ $\overline{<T>}^{1 / 8 \chi \pi \alpha_{2}}$. Considering $\chi<0$ indicates that decreasing $\overline{\langle T\rangle}$ results in increasing the rupture velocity (i.e., $\overline{<T>} \propto \Xi^{\chi}$ in parameter space).

The obtained result is comparable with the results of several investigations regarding the weak increase of $\Gamma$ with rupture velocity (Freund, 1979; Livne et al., 2008). Note that this conclusion is based on the assumption of constant stress drop which corresponds to a constant value for $\Delta Q=Q_{\max }-Q_{\min }$. We also can extend the concept of the rate of energy dissipation to the "slow class" regime (R3) to study how fracture energy changes in the slow-slip regime. In this case, $\chi>0$ and decreasing $\overline{\langle T\rangle}$ yields decreasing $\Gamma$. In other words, far from the transition point (from

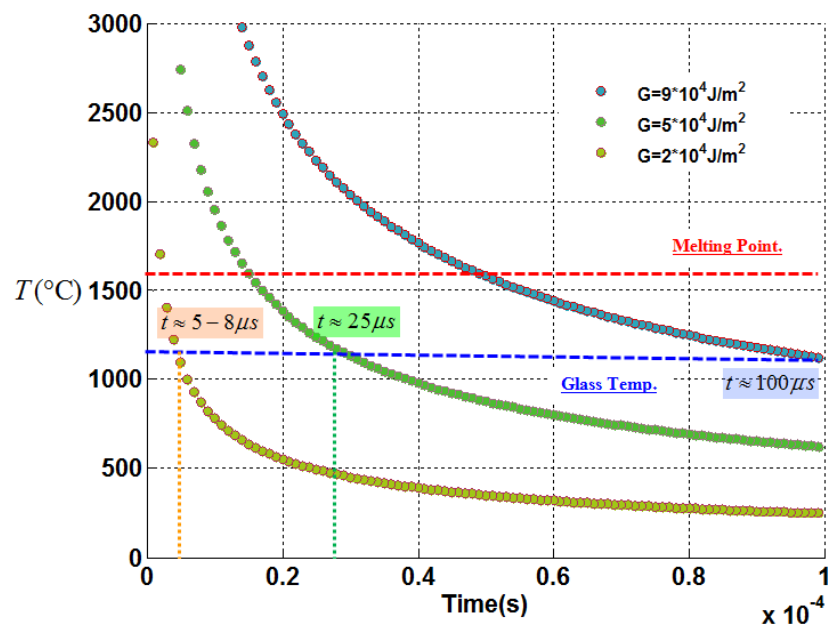

Figure 6. Transition to the "rubber-like" phase using a 1-D heat-diffusion model for a submicron process zone. For $G \approx 5 \times$ $10^{4} \mathrm{~J} \mathrm{~m}^{-2}$ (Lockner et al., 1991), the duration of the second phase $t \approx 25 \mu$ s is satisfied.

the $\mathrm{R} 3$ to the $\mathrm{R} 2$ class) and in the slow-rupture class, the rate of energy dissipation is low. The latter conclusion has been proven numerically in Braun et al. (2009) and recently in Bouchbinder et al. (2011) through spring-block models. In Fig. 6, we show how the temperature of the process zone at submicron length scale can rise above the melting temperature and the glass point of $\mathrm{SiO}_{2}$. The employed fracture energy remarkably agrees with the values reported in Lockner et al. (1991).

To confirm the nature of amplified events and their relations with our formulation, we tested some tiny events from two well-studied experiments. The first experiment was a double friction test including gouge materials sheared between two steel plates (Mair et al., 2007). Events emitted 

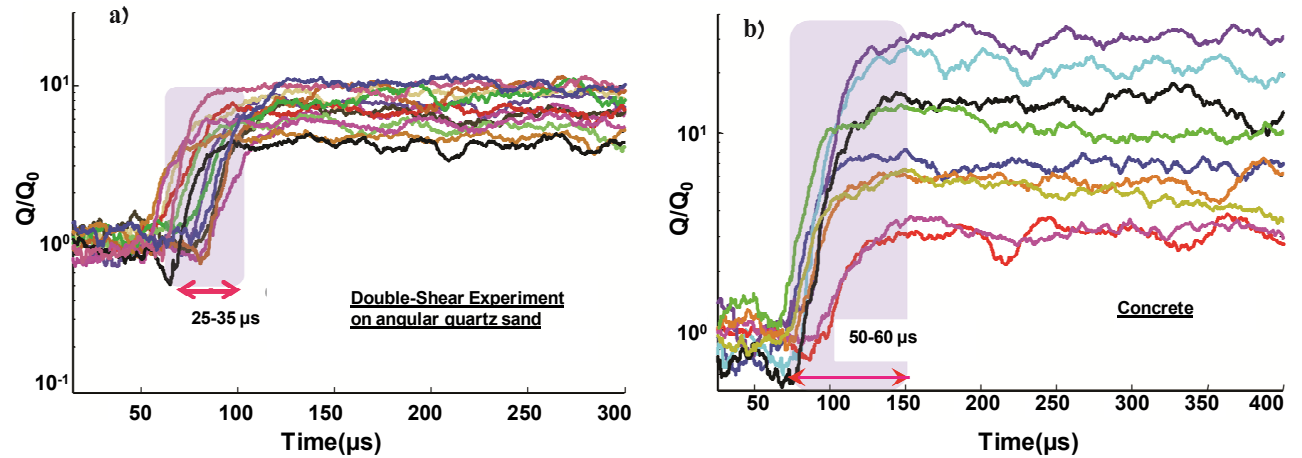

Figure 7. Duration of the second phase in two different experiments: (a) double shear text as reported in Mair et al. (2007), where quartzgouge materials are sheared between two steel plates. The duration of the second phase is about 25-30 $\mu$ s. (b) Waveforms from concrete samples as reported in Katsaga et al. (2007) show a longer fast-slip phase. This is due to the nature of C-S-H bands, and the microcracks involve bond breaking and bond rearrangements at the crack tip (Buehler and Keten, 2010).

from angular quartz sand reflect a similar fast-slip phase duration to Lab.Eq.1 and Lab.Eq.2 (Fig. 7a). The second additional experiment used concrete samples (mainly Portland cement) (Katsaga et al., 2007). The results reflect about $\sim 55-60 \mu$ s duration of the fast-slip period, 2 to 3 times longer than in other silicate-based rock tests (Fig. 7b). Considering the fact that the toughness of concrete is higher than for silicate-based rocks $\left(\mathrm{SiO}_{2}\right.$ is the dominant mineral in granite, basalt and some sandstones), we assign this feature to a longer fast-slip stage. This can also be due to additional sources of energy propagation involved in colloidal systems (such as cement and some polymers) as they include bond breaking and bond rearrangements at the crack tip (Buehler and Keten, 2010). Then, our effective temperature model successfully matches with the released energy during microcracking, well coupled with the details of cracking bonds and other additional sources of energy dissipation at the molecular/atomic levels. In summary, we speculate that materials with higher plasticity (and thus high resistance against cracking) imprint a relatively longer fast-weakening stage.

\section{Conclusions}

In this study, we presented novel results on a proposed approach of functional friction networks. The results suggest that the micron (and submicron) rupture fronts imprint critical scaling exponents in various scalar network parameters, comparable with the possible slow and regular deformation or "earthquakes". We showed that the complex shape of waveforms during cracking reflects a multiscale physical process involving the onset of cracking, microsecondsscale initial strengthening or microdynamic loading, fastweakening, re-embrittlement and healing phases.

Our method is the first known network-based algorithm that uncovers such details of crackling. For the first time, we observed that the initial strengthening phase for most of the events exhibits a constant timescale, which suggests the possibility of the thermally activated nature of submicron instability in the tip of moving crack tips. Moreover, we formulated the energy of the networks (and thus the kinematic energy of the system) based on the rate of change of the modularity. This enabled the inference of a possible relationship between the remote stress field as the driving field and the temporal average modularity comparable with the quantified experimental results. As a result of our novel formulation, a singularity in the transition from slow ruptures to the subRayleigh class (or vice versa) was found which is compatible with the recent direct observation of rupture transitions in glassy materials.

In fact, our results regarding the classification of microevents are in full agreement with the rate of the fastweakening stage (rate of fast decrease of shear stress in the course of weakening): the faster-weakening phase correlates with fast-rupture velocity. This scenario is possible in two ways: shorter weakening time and/or stronger stress drop. From another perspective, we speculated that the duration of the fast-slip stage is nearly the same for the most dominant events. We provided a simple explanation for this invariant signature using an effective temperature model previously applied to PMMA (Ben-David et al., 2010b, c), which illuminates the role of fracture surface energy in the duration of this phase. The rate of immediate healing after the peak of $Q(t)$ is also explained with this simple model. However, some questions need to be investigated in more details. Among them, one might be interested in the details of the third phase (sudden healing), where the rate of healing equals that of the fastweakening period or is even higher than during that stage. In this case, we will deal with pulse-like ruptures which imprint nearly symmetric pulse shapes. Yet another interesting aspect would involve investigating the details of the evolution of the fast-weakening stage. We expect to see some nonlinearity in this period due to the effect of hyperelasticity at the crack tip as well as ductile cracking. Another tempting aspect would involve investigating the few-tens-of-nanoseconds-resolution 
data of the initial strengthening stage. The idea is that, assuming a maximum strength of an asperity (or a single contact) of $\sim \mathrm{MPa}$ and loading time $\sim \mu$ s, we reach a few hundred $\mathrm{GPa} / \mathrm{s}$ loading rate. This loading rate is enough to pulverize the asperity, which produces a few micrometer-sized gouge particles. Under this extreme rise of load - which occurs near a moving crack tip - real solids show strong nonlinearity in bulk modulus parameters. It would be interesting to examine how the initial phase likely affects the second and other phases.
In summary, this work demonstrates that network theory has a great potential to analyze and extract new information from laboratory experiments featuring full waveform AEs. The interpretation of network parameters in light of nearly constant evolutionary phases has the potential to be a very useful indicator and provide new insights into the evolution of rupture tips. The techniques and approaches described here have significant potential in terms of their application to $\mathrm{AE}$ events originating from conditions such as complex boundary conditions, fluid-driven fractures, noise coupled processes such as thermal-mechanical cracks, events from high temperature and high pressure (multi-anvil tests) and crackling noises from sheared granular materials or dislocations. Furthermore, from a network science perspective, this research featured relatively simple algorithms for the construction of the functional networks. Future approaches could include the use of more sophisticated, weighted networks and directed graph theory to better elucidate the behavioral processes and transitions from micro- to macroscale fractures. 


\section{Appendix A}

Table A1. List of parameters used in this study.

\begin{tabular}{|c|c|}
\hline Parameter & Description \\
\hline$k_{i}$ & Degree (number of links) of node $i$ \\
\hline$C$ & Global clustering coefficient \\
\hline$\Sigma$ & Skewness of modularity profiles \\
\hline$\overline{<T>}$ & $\begin{array}{l}\text { Spatiotemporal mean of the number of triangles attached to each vertex. Spatial average is on all nodes } \\
\text { and temporal mean is on the short-term monitored interval. }\end{array}$ \\
\hline$\overline{<\mathrm{B} . \mathrm{C}>}$ & Spatiotemporal average of betweenness \\
\hline$Q_{\max }$ & Maximum modularity value in the time interval in which the evolution of waveforms is monitored \\
\hline$\Xi$ & $\Xi \equiv \overline{\log <\mathrm{B} . \mathrm{C}>}^{-1}$ \\
\hline$\dot{Q}_{i}$ & The rate of $Q(t)$ for each generic phase in the $Q$ profiles \\
\hline$R$ & $\begin{array}{l}R \text { profiles as the reciprocal of } Q \text { profiles. We use this value to place emphasis on the first evolutionary } \\
\text { phase as the nucleation and main deformation phase of microcracks. }\end{array}$ \\
\hline$\chi$ & Power exponent in $\overline{<T>} \propto \overline{\log <\text { B.C }>}-\chi$ \\
\hline$\alpha_{2}$ & Power exponent in $\dot{Q}_{\mathrm{II}} \propto Q_{\max }^{\alpha_{2}}$ \\
\hline$\pi$ & Power exponent in $Q_{\max } \propto \Xi^{-\pi}$ \\
\hline$\lambda$ & Power exponent in $\left|\dot{Q}_{\mathrm{IV}}\right| \propto Q_{\max }^{\lambda}$ \\
\hline$\gamma$ & Power exponent in $\left|\dot{Q}_{\mathrm{IV}}\right| \propto\left|\dot{Q}_{\mathrm{I}}\right|^{-\gamma}$ \\
\hline$\mu$ & Power exponent in $\dot{R} \propto R_{\max }^{\mu}$ \\
\hline$E$ & Sum of energy of the generic phases in $Q$ profiles \\
\hline$\kappa$ & Coefficient in $\bar{Q} \propto \exp \left(-\kappa F_{\text {axial }}\right)$ \\
\hline$\eta$ & Power exponent in $\bar{Q} \propto \Xi^{-\eta}$ \\
\hline$F_{\text {axial }}$ & External axial load as the driving force of the system \\
\hline$\Gamma$ & Fracture surface energy: onset of cracking is satisfied when fracture energy meets Griffiths' crack criterion. \\
\hline
\end{tabular}


Acknowledgements. We would like to acknowledge and thank D. Lockner (USGS, Menlo-Park, USA), S. Nielsen (Istituto Nazionale di Geofisica e Vulcanologia, Italy), T. Katsaga (Itasca, Toronto), K. Mair (Physics of Geological Processes, University of Oslo, Oslo, Norway) and C. Marone (Pennsylvania State University, University Park, Pennsylvania, USA) for providing the data set employed in this work. Reviewers' comments helped to increase the quality of the initial manuscript and are gratefully acknowledged. The first author would like to acknowledge comments and points from Reik Donner.

Edited by: R. Donner

Reviewed by: four anonymous referees

\section{References}

Alon, U.: Network motifs: theory and experimental approaches, Nat. Rev. Genet., 8, 450-461, 2007.

Andrews, D. J.: Rupture velocity of plane strain shear cracks, J. Geophys. Res. B, 81, 5679-5687, 1976.

Andrews, D. J.: Dynamic plane-strain shear rupture with a slipweakening friction law calculated by a boundary integral method, B. Seismol. Soc. Am., 75, 1-21, 1985.

Arnhold, J., Grassberger, P., Lehnertz, K., and Elger, C. E.: A robust method for detecting interdependences: application to intracranially recorded EEG, Physica D, 134, 419-430, 1999.

Bashan, A., Bartsch, R. P., Kantelhardt, J. W., Havlin, S., and Ivanov, P. C.: Network physiology reveals relations between network topology and physiological function, Nat. Commun., 3, 19, 2012.

Bassett, D. S., Meyer-Lindenberg, A., Achard, S., Duke, T., and Bullmore, E.: Adaptive reconfiguration of fractal small-world human brain functional networks, Proc. Natl. Acad. Sci., 103, 19518-19523, 2006.

Ben-David, O. Cohen, G., and Fineberg, J.: The dynamics of the onset of frictional slip, Science, 330, 211-214, 2010a.

Ben-David, O., Rubinstein, S., and Fineberg, J.: Slip-Stick: The evolution of frictional strength, Nature, 463, 76-79, 2010b.

Ben-David, O., Cohen, G., and Fineberg, J.: Short-time dynamics of frictional strength in dry friction, Tribol. Lett., 39, 235-245, 2010c.

Blondel, V. D., Guillaume, J. L., Lambiotte, R., and Lefebvre, E.: Fast unfolding of communities in large networks, J. Stat. Mechan. Theor. Experim., 10, P10008, doi:10.1088/17425468/2008/10/P10008, 2008.

Bouchbinder, E., Brener, E. A., Barel, I., and Urbakh, M.: Dynamics at the onset of frictional sliding, Phys. Rev. Lett., 107, 235501, doi:10.1103/PhysRevLett.107.235501, 2011.

Braun, O. M., Barel, I., and Urbakh, M. :Dynamics of transition from static to kinetic friction, Phys. Rev. Lett., 103, 194301, doi:10.1103/PhysRevLett.103.194301, 2009.

Buehler, M. J. and Keten, S.: Colloquium: Failure of molecules, bones, and the Earth itself, Rev. Modern Phys., 82, 1459-1487, 2010.

Chen, Y., Rangarajan, G., Feng, J., and Ding, M.: Analyzing multiple nonlinear time series with extended Granger causality, Phys. Lett. A, 324, 26-35, 2004.
Donges, J. F., Zou, Y., Marwan, N., and Kurths, J.: The backbone of the climate network, Europhys. Lett., 87, 48007, doi:10.1209/0295-5075/87/48007, 2009.

Donner, R. V., Zou, Y., Donges, J. F., Marwan, N., and Kurths, J.: Recurrence networks - A novel paradigm for nonlinear time series analysis, New J. Phys., 12, 033025, doi:10.1088/13672630/12/3/033025, 2010.

Dunham, E. M.: Conditions governing the occurrence of supershear ruptures under slip-weakening friction, J. Geophys. Res., 112, B07302, doi:10.1029/2006JB004717, 2007.

Freund, L. B.: The mechanics of dynamic shear crack propagation, J. Geophys. Res., 84, 2199-2209, 1979.

Ghaffari, H. O. and Young, R. P. : Network configurations of dynamic friction patterns, EPL, 98, 48003, doi:10.1209/02955075/98/48003, 2012a.

Ghaffari, H. O. and Young, R. P.: Topological complexity of frictional interfaces: friction networks, Nonlin. Processes Geophys., 19, 215-225, doi:10.5194/npg-19-215-2012, 2012 b.

Ghaffari, H. O. and Young, R. P.: Acoustic-friction networks and the evolution of precursor rupture fronts in laboratory earthquakes, Sci. Reports 3, 1799, doi:10.1038/srep01799, 2013.

Ghaffari, H. O., Sharifzadeh, M., and Young, R. P.: Complex aperture networks, Physica A: Statistical Mechanics and its Applications, 392, 1028-1037, 2013.

Ghaffari, H. O., Nasseri, M. H. B., and Young, R. P.: Faulting of Rocks in a Three-Dimensional Stress Field by Micro-Anticracks, Sci. Reports, 4, 5011, doi:10.1038/srep05011, 2014.

Guimerà, R. and Amaral, L. A. N.: Functional cartography of complex metabolic networks, Nature, 433, 895-900, 2005.

Iwayama, K., Hirata, Y., Takahashi, K., Watanabe, K., Aihara, K., and Suzuki, H.: Characterizing global evolutions of complex systems via intermediate network representations, Sci. Reports 2, 423, doi:10.1038/srep00423, 2012.

Kaneko, Y. and Ampuero, J. P.: A mechanism for preseismic steady rupture fronts observed in laboratory experiments, Geophys. Res. Lett., 38, L21307, doi:10.1029/2011GL049953, 2011.

Katsaga, T., Sherwood, E. G., Collins, M. P., and Young, R. P.: Acoustic emission imaging of shear failure in large reinforced concrete structures, Int. J. Fracture, 148, 29-45, 2007.

Li, C.-B., Yang, H., and Komatsuzaki, T.: Multiscale complex network of protein conformational fluctuations in single-molecule time series. Proc. Natl. Acad. Sci., 105, 536-541, 2008.

Livne, A., Bouchbinder, E., and Fineberg, J.: The breakdown of linear elastic fracture mechanics near the tip of a rapid crack, Phys. Rev. Lett., 101, 264301, doi:10.1103/PhysRevLett.101.264301, 2008.

Lockner, D. A., Byerlee, J. D., Kuksenko, V., Ponomarev, A., and Sidorin, A.: Quasi-static fault growth and shear fracture energy in granite, Nature, 350, 39-42, 1991.

Maercklin, N., Festa, G., Colombelli, S., and Zollo, A.: Twin ruptures grew to build up the giant 2011 Tohoku, Japan, earthquake, Sci. Reports, 2, 709, doi:10.1038/srep00709, 2012.

Mair, K., Marone, C., and Young, R. P.: Rate dependence of acoustic emissions generated during shear of simulated fault gouge, B. Seismol. Soc. Am., 97, 1841-1849, 2007.

Mehta, A. P., Mills, A. C., Dahmen, K. A., and Sethna, J. P.: Universal pulse shape scaling function and exponents: Critical test for avalanche models applied to Barkhausen noise, Phys. Rev. E, 65, 046139, doi:10.1103/PhysRevE.65.046139, 2002. 
Meng, L. Inbal, A., and Ampuero, J.-P.: A window into the complexity of the dynamic rupture of the $2011 \mathrm{Mw} 9$ Tohoku-Oki earthquake, Geophys. Res. Lett., 38, L00G07, doi:10.1029/2011GL048118, 2011.

Milo, R., Shen-Orr, S., Itzkovitz, S., Kashtan, N., Chklovskii, D., and Alon, U.: Network motifs: simple building blocks of complex networks, Science, 298, 824-827, 2002.

Napoletani, D. and Sauer, T.: Reconstructing the topology of sparsely connected dynamical networks, Phys. Rev. E, 77, 026103, doi:10.1103/PhysRevE.77.026103, 2008.

Newman, M. E. J.: Networks: An Introduction, Oxford University Press, 2010.

Newman, M. E. J. and Girvan, M.: Finding and evaluating community structure in networks, Phys. Rev. E, 69, 026113, doi:10.1103/PhysRevE.69.026113, 2004.

Nielsen, S., Taddeucci, J., and Vinciguerra, S.: Experimental observation of stick-slip instability fronts, Geophys. J. Int., 180, 697702, doi:10.1111/j.1365-246X.2009.04444.x, 2010.

Noda, H. and Lapusta, N.: Stable creeping fault segments can become destructive as a result of dynamic weakening, Nature, 493, 518-521, 2013.

Palla, G., Derényi, I., Farkas, I., and Vicsek, T.: Statistical mechanics of topological phase transitions in networks, Phys. Rev. E, 69, 046117, doi:10.1103/PhysRevE.69.046117, 2004.

Rice, J. R., Lapusta, N., and Ranjith, K.: Rate and state dependent friction and the stability of sliding between elastically deformable solids, J. Mech. Phys. Solids, 49, 1865-1898, 2001.

Rubinstein, S., Cohen, G., and Fineberg, J.: Detachment fronts and the onset of dynamic friction, Nature, 430, 1005-1009, 2004.
Rycroft, C. H. and Bouchbinder, E.: Fracture Toughness of Metallic Glasses: Annealing-Induced Embrittlement, Phys. Rev. Lett., 109, 194301, doi:10.1103/PhysRevLett.109.194301, 2012.

Schiff, S. J., So, P., Chang, T., Burke, R. E., and Sauer, T.: Detecting dynamical interdependence and generalized synchrony through mutual prediction in a neural ensemble, Phys. Rev. E, 54, 67086724, 1996.

Segall, P., Rubin, A. M., Bradley, A. M., and Rice, J. R.: Dilatant strengthening as a mechanism for slow slip events, J. Geophys. Res., 115, B12305, doi:10.1029/2010JB007449, 2010.

Simons, M., Minson, S. E., Sladen, A., Ortega, F., Jiang, J., Owen, S. E., and Webb, F. H.: The 2011 magnitude 9.0 Tohoku-Oki earthquake: Mosaicking the megathrust from seconds to centuries, Science, 332, 1421-1425, 2011.

Sone, H. and Shimamoto, T.: Frictional resistance of faults during accelerating and decelerating earthquake slip, Nat. Geosci., 2, 705-708, 2009.

Thompson, B. D., Young, R. P., and Lockner, D. A.: Observations of premonitory acoustic emission and slip nucleation during a stick slip experiment in smooth faulted Westerly granite, Geophys. Res. Lett., 32, L10304, doi:10.1029/2005GL022750, 2005.

Thompson, B. D., Young, R. P., and Lockner, D. A.: Premonitory acoustic emissions and stick-slip in natural and smoothfaulted Westerly granite, J. Geophys. Res., 114, B02205J, doi:10.1029/2008JB005753, 2009.

$\mathrm{Xu}, \mathrm{X}$., Zhang, J., and Small, M.: Superfamily phenomena and motifs of networks induced from time series, Proc. Nalt. Acad. Sci., 105, 19601-19605, 2008.

Zapperi, S., Castellano, C., Colaiori, F., and Durin, G.: Signature of effective mass in crackling-noise asymmetry, Nat. Phys., 1, 46-49, 2005. 